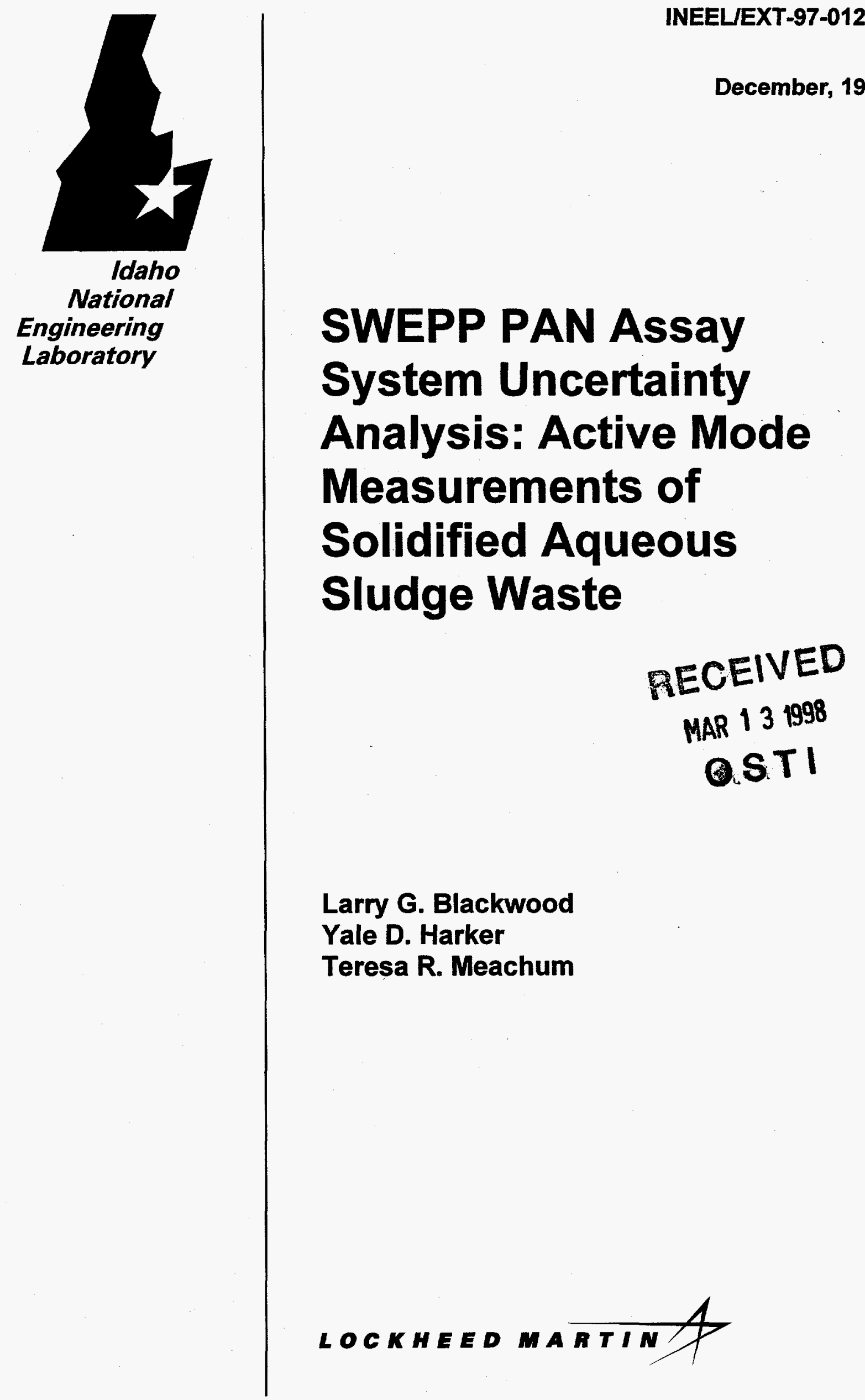




\title{
SWEPP PAN Assay System Uncertainty Analysis: Active Mode Measurements of Solidified Aqueous Sludge Waste
}

\author{
Larry G. Blackwood \\ Yale D. Harker \\ Teresa R. Meachum
}

Published December 1997
Idaho National Engineering and Environmental Laboratory TRU Waste Programs
Lockheed Martin Idaho Technologies
Idaho Falls, Idaho 83415

\author{
Prepared for the \\ U. S. Department of Energy \\ Office of Environmental Restoration and Waste Management \\ Under DOE Idaho Operations Office \\ Contract DE-AC07-94ID13223
}




\section{DISCLAIMER}

This report was prepared as an account of work sponsored by an agency of the United States Government. Neither the United States Government nor any agency thereof, nor any of their employees, makes any warranty, express or implied, or assumes any legal liability or responsibility for the accuracy, completeness, or usefulness of any information, apparatus, product, or process disclosed, or represents that its use would not infringe privately owned rights. Reference herein to any specific commercial product, process, or service by trade name, trademark, manufacturer, or otherwise does not necessarily constitute or imply its endorsement, recommendation, or favoring by the United States Government or any agency thereof. The views and opinions of authors expressed herein do not necessarily state or reflect those of the United States Government or any agency thereof. 


\section{DISCLAIMER}

Portions of this document may be illegible electronic image products. Images are produced from the best available original document. 


\begin{abstract}
The Idaho National Engineering and Environmental Laboratory is being used as a temporary storage facility for transuranic waste generated by the U. S. Nuclear Weapons program at the Rocky Flats Plant (RFP) in Golden, Colorado. Currently, there is a large effort in progress to prepare to ship this waste to the Waste Isolation Pilot Plant (WIPP) in Carlsbad, New Mexico. In order to meet the TRU Waste Characterization Quality Assurance Program Plan nondestructive assay compliance requirements and quality assurance objectives, it is necessary to determine the total uncertainty of the radioassay results produced by the Stored Waste Examination Pilot Plant (SWEPP) Passive Active Neutron (PAN) radioassay system. This paper is one of a series of reports quantifying the results of the uncertainty analysis of the PAN system measurements for specific waste types and measurement modes. In particular this report covers active mode measurements of weapons grade plutonium-contaminated aqueous sludge waste contained in 208 liter drums (item description codes 1, 2, 7, 800, 803, and 807).
\end{abstract}

Results of the uncertainty analysis for PAN active mode measurements of aqueous sludge indicate that a bias correction multiplier of 1.55 should be applied to the PAN aqueous sludge measurements. With the bias correction, the uncertainty bounds on the expected bias are $0 \pm 27 \%$. These bounds meet the Quality Assurance Program Plan requirements for radioassay systems. 


\section{SUMMARY}

This report describes the results of a total uncertainty analysis of the PAN system's active mode measurements of aqueous sludge waste (item description codes 1, 2, 7, 800, 803, and 807). The uncertainty analysis was performed by comparing PAN system active mode results to radiochemistry results for 125 aqueous sludge drums. The radiochemistry data were obtained from core samples of the waste drums. Bias in the PAN system was estimated by regression of the radiochemistry results (assumed to be unbiased) on the PAN measurements. Precision of the PAN measurements was assessed by examination of the variance components associated with the regression.

Analysis of the results for the 125 aqueous waste drums indicates that a bias correction multiplier of 1.55 should be applied to the PAN aqueous sludge measurements. Without this correction, the PAN measurements are biased low by $35 \%$ relative to the radiochemistry measurements. With the bias correction, the uncertainty bounds on the expected bias are $0 \pm 27 \%$. These bounds meet the Quality Assurance Program Plan requirements for radioassay systems.

The total uncertainty (expressed as a relative standard deviation) for the PAN measurements was found to be approximately $53 \%$, based on a regression analysis. However, the median value for the relative total uncertainty in the 125 cases was only $30 \%$.

In order to be critically safe, there is a safety limit requiring that the measured Pu mass plus 2 times its standard error must not exceed $200 \mathrm{~g}$. Based on the average total uncertainty calculations derived in this report the measured mass must be below approximately $97 \mathrm{~g}$ in order to meet this criterion for drums containing aqueous sludge waste. However, if the median total uncertainty value is used, the limit is increased to $125 \mathrm{~g}$. These summary mass values are only rough indicators of limits on shippable quantities, as the actual upper limits will be calculated individually for each drum. In any case, the $200 \mathrm{~g}$ shipping limit is not likely to be an issue for sludge wastes as these drums typically contain only small Pu quantities (i.e., $<10 \mathrm{~g}$ ). 


\section{ACKNOWLEDGMENTS}

The authors would like to acknowledge the support of others who have made significant contributions to the production of this report. Dr. L. V. East and C. R. Hoffman developed the data files and data listings containing the results of the PAN measurements pertaining to the aqueous sludge measurements. Dr. $\mathrm{L}$. A. Van Ausdeln and C. V. McIsaac served as technical reviewers. G. Twedell is the physics support technical leader at SWEPP and the authors would like to thank him for his assistance in obtaining the PAN assay data used in this project. D. E. Menkhaus is the project sponsor and the authors would like to thank him for his support and encouragement during the course of this evaluation.

This document was prepared for the U. S. Department of Energy Office of Environmental Restoration and Waste Management under DOE Idaho Operations Office Contract DE-AC07-94ID13223. 


\section{CONTENTS}

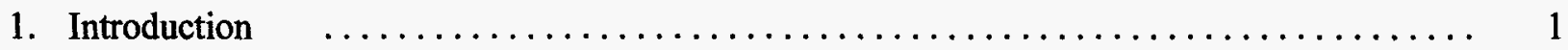

2. Passive Active Neutron (PAN) Assay System $\quad \ldots \ldots \ldots \ldots \ldots \ldots \ldots \ldots \ldots \ldots \ldots$

3. Calibration of the PAN Assay System $\quad \ldots \ldots \ldots \ldots \ldots \ldots \ldots \ldots \ldots \ldots \ldots \ldots \ldots \ldots$

4. Contributors to the Assay Uncertainties $\quad \ldots \ldots \ldots \ldots \ldots \ldots \ldots \ldots \ldots \ldots \ldots \ldots$

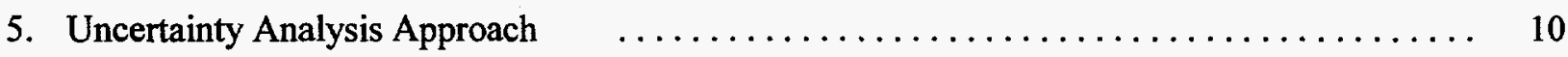

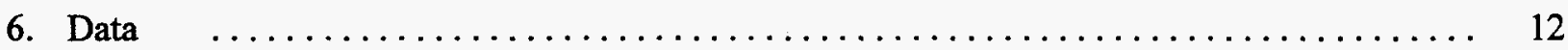

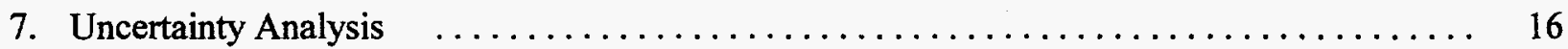

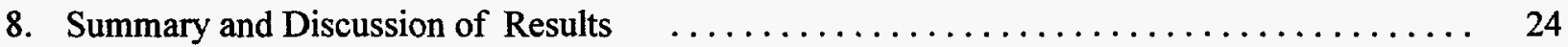

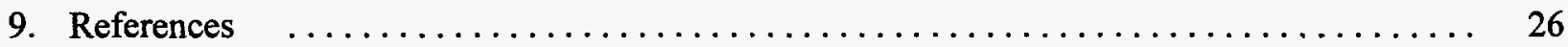

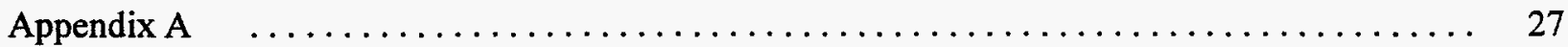

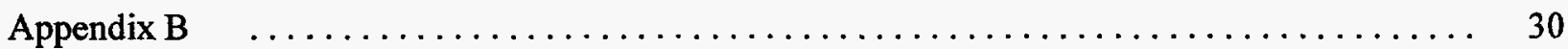




\section{SWEPP PAN Assay System Uncertainty Analysis: Active Mode Measurements of Solidified Aqueous Sludge Waste}

\section{INTRODUCTION}

The Idaho National Engineering and Environmental Laboratory is being used as a temporary storage facility for transuranic waste generated by the U. S. Nuclear Weapons program at the Rocky Flats Plant (RFP) in Golden, Colorado. Currently, there is a large effort in progress to prepare to ship this waste to the Waste Isolation Pilot Plant (WIPP) in Carlsbad, New Mexico. In order to meet the TRU Waste Characterization Quality Assurance Program Plan nondestructive assay compliance requirements and quality assurance objectives, it is necessary to determine the total uncertainty of the radioassay results produced by the Stored Waste Examination Pilot Plant (SWEPP) Passive Active Neutron (PAN) radioassay system. This paper is one of a series of reports quantifying the results of the uncertainty analysis of the PAN system measurements for specific waste types and measurement modes. In particular this report covers PAN active mode measurements of weapons grade plutonium-contaminated aqueous sludge waste contained in 208 liter drums (item description codes 1, 2, 7, 800, 803, and 807).

The PAN assay system itself calculates uncertainty based on the counting statistics of the assay technique. However, depending on the waste form involved, there can be significant additional systematic and random errors that need to be quantified before the total assay uncertainty can be quoted. To estimate the magnitude of the additional effects a sampling and verification approach was used, i.e., a sample of PAN measurements were compared to confirmatory measurements on an alternative measurement system. For the aqueous sludge wastes, the confirmatory measurements consisted of radiochemistry data. The radiochemistry data were obtained from core samples of the waste drums. Bias in the PAN system was estimated by regression of the radiochemistry results (assumed to be unbiased) on the PAN measurements. Precision of the PAN measurements was assessed by examination of the variance components associated with the regression. Because the active mode result is always selected as the reported mass for sludge wastes, this report only addresses uncertainty in the active mode measurements.

Sections 2, 3, and 4 of this report give a general description of the PAN assay system and operation (in particular the active mode) and potential sources of uncertainty in the measurement. The uncertainty analysis approach is described in detail in Section 5. Section 6 discusses the data used in support of the uncertainty analysis. Results of the analysis are presented in Sections 7 and 8. 


\section{PASSIVE ACTIVE NEUTRON (PAN) ASSAY SYSTEM}

The SWEPP PAN system is a second generation passive active neutron system developed in the early 1980 's by Los Alamos National Laboratory for the U. S. Department of Energy (DOE) and delivered to the Idaho National Engineering and Environmental Laboratory (INEEL) in 1983. This systern was designed to assay drums containing transuranic contaminated waste. The SWEPP PAN assay system is described in an INEEL internal document (Becker, 1993); for more details the reader is referred to that document. A summary description is given below.

The PAN assay system consists of a shielding housing which surrounds the drum on all four sides, top and bottom. Each side of the housing contains moderator (i.e., graphite, polyethylene), thermal and lowenergy neutron shielding (i.e., cadmium, boron) and He-3 neutron detectors. There are two types of detector assemblies contained in each side of the assay system; i.e., bare detectors and shielded detectors.

The shielded detectors are grouped into detector packages where each package is surrounded by a thermal and low-energy neutron shield consisting of cadmium and borated rubber. Inside the cadmium and borated rubber are three or four He-3 neutron detectors surrounded by polyethylene. This type of detector assembly is sensitive to fast neutrons and insensitive to thermal and low-energy neutrons. The bare detectors are also He-3 detectors surrounded by polyethylene but are not shielded by cadmium or borated rubber. In this configuration they are sensitive to all neutrons.

The assay system operates in two modes, passive and active. In the passive mode the detector assernblies (bare and shielded) are detecting neutrons produced by spontaneous fission and $(\alpha, n)$ interactions in the waste matrix. Differentiation between the fission neutrons and the $(\alpha, n)$ neutrons is accomplished by coincidence event counting. In this type of counting, a coincidence event is recorded when two neutrons are detected by the system within a specified time window. There are two coincidence windows used: one is $35 \mu \mathrm{s}$ long and looks for coincidence events from the shielded detectors in the enclosure. This is referred to as the short-gate (SG) or shielded coincidence mode. The other gate is $250 \mu \mathrm{s}$ long and looks for coincidence events from all "system" detectors (shielded and bare) in the enclosure. There are two coincidence circuits that use all system detectors and a $250 \mu$ s gate window. The first circuit is referred as the long-gate (LG) or system coincidence mode. The second circuit is the shift register coincidence (SRC) unit.

In addition to the coincidence counting, single event counting is also accumulated during the passive mode. The single event counting data are used to derive chance coincidence corrections to the coincidence data and also to arrive at a moderator index (MI).

In the active mode the shielded detectors are used to detect neutrons produced by stimulated fission resulting from thermal neutron interrogation. The interrogation neutron source for the active mode is a Zetatron $14 \mathrm{MeV}$ neutron generator located at one corner inside the system shield enclosure. The high energy neutrons are moderated to thermal via the graphite moderator in the enclosure walls and varying amounts of moderator in the waste matrix. For the active mode the signal of interest is taken from a gated count of the shielded detectors for the time window from $700 \mu \mathrm{s}$ to $2700 \mu \mathrm{s}$ following each neutron burst from the neutron generator. This time window was selected to allow the fast neutrons from the generator to thermalize in the enclosure and thereby have a higher probability to stimulate fission in Pu-239 and at the same time the thermalized interrogation neutrons are not detectable by the shielded detectors. To account for background, another count window is opened from $5700 \mu$ s to $15700 \mu$ s after each neutron burst. It is expected that during this time window only background neutrons will be present. 
Also during the active mode two monitors are used to monitor the interrogation neutron flux and the effective transmission of interrogation neutrons through the contents of the drum. The first monitor, called the cavity monitor, consists of one bare $\mathrm{He}-3$ detector mounted inside the assay system enclosure on the back wall of the chamber along an upper corner. The second monitor, called the barrel flux monitor, is a single He-3 detector inside a cadmium collimator mounted at mid-height in the adjacent back corner of the assay system enclosure so that the detector's field of view is the center of the drum. These two monitors are operated with the same time windows as the shielded detectors during the active mode. The ratio of the cavity monitor count during active mode to the barrel monitor count during active mode is referred to as the absorber index (AI).

The moderator index (from passive mode counts) and the absorber index (from the active mode count) are used in the analysis algorithm to arrive at correction factors which are intended to correct for moderator and absorber effects on the measured responses (both active and passive responses). The corrected responses are used to determine the measured plutonium mass. Therefore, both the active and passive counts must be completed to obtain the needed correction factors. Four measured mass values are obtained by the system for each measurement sequence (passive count + active count). That is, there is a mass value determined from the active mode count, a mass value determined from the passive short-gate coincidence count, a mass value determined from the passive long-gate coincidence count, and a mass value determined from the shift register coincidence count. However, not all four values are valid over the mass range and waste forms covered in SWEPP waste. A set of selection algorithms is included in the system software to determine which of the four assay values should be used in the waste certification documentation.

\subsection{Assay of Sludge Waste Forms Using the PAN System}

Sludge is a general term referring to solidified process waste. Under this general category there are several Item Description Codes (IDCs); e.g., 1, 2, 3, 7, 92, 800, 801, 802, 803, 806, 807, etc. The subdivision into IDC designations is based on the process from which the waste was generated and the treatment and packaging of the waste after generation. Generally speaking there is no evidence of any clumping of plutonium into chunks large enough to create significant self-shielding. The major difficulty concerning assay using passive coincidence methods on sludge waste drums is high $(\alpha, n)$ neutron backgrounds present in most sludge drums. Because of high $(\alpha, n)$ background and relatively low plutonium content, the passive mode was considered to lack the sensitivity or accuracy to assay sludge waste forms. However, this assessment was done prior to the installation of the shift register coincidence unit. This new unit has virtually removed $(\alpha, n)$ backgrounds as a reason to exclude passive techniques; however, passive techniques still lack the sensitivity to assay plutonium in those cases where it is less than $5 g$.

By default, the active mode became the mode of choice for reporting plutonium mass in sludge waste. The relatively high density and hydrogen content do pose problems concerning interrogation neutron penetration into the center of a drum and do affect the overall accuracy of the active mode when assaying sludge waste. Early studies have indicated that the bias and precision of the active mode results were suspect. For example, a previous assessment was conducted where radiochemistry data on samples taken from 24 sludge drums were analyzed by Oak Ridge National Laboratory and compared with early PAN assay data for the same set of drums. Because of incomplete data and low sample population, the results from this assessment were not formally reported. However, they did indicate that the active assay results from early PAN assays were low by a factor of 2.2 and that the relative precision error was about $40 \%$. 


\subsection{Active Mode Response}

The interrogation/counting sequence used in the PAN active mode assay is commonly referred to as the "Differential Die-away Technique" (DDT). In the active mode assay, the PAN neutron generator is operated at a rate of 50 pulses per second. At this repetition rate and with $\approx 2 \times 10^{6}$ interrogation neutrons per pulse, the output from the neutron generator is $\approx 10^{8}$ neutrons per second . Currently an active mode data assay will use 4000 neutron generator pulses, which takes about 80 real time seconds to complete.

There are three detector systems used during active mode interrogation:

1. shielded detector banks in walls, door, top and floor of enclosure,

2. cavity flux monitor mounted in an upper corner of the assay chamber, and

3. barrel flux monitor with collimator shield mounted in the cavity at drum mid-height.

Each shielded detector bank, cavity flux monitor and barrel flux monitor has two count registers, one accumulates detector counts arriving during the early gate time window and the other accumulates detector counts arriving during the late gate time window. For total plutonium analysis, the individual detector bank count data are summed to produce a shielded total count for the whole system. In the active mode assay, there is one shielded total count for the early gate time window and one shielded total count for the late gate time window.

The basic active mode signal is derived from shielded detector counts produced by fission neutrons arising from thermal neutron stimulated fission in Pu-239 or other fissile nuclides. The purpose of the neutron generator is to produce a source of neutrons that will produce fission. However, the neutron generator produces high-energy neutrons $(14 \mathrm{MeV})$ whose fission cross section for Pu-239 is relatively low $(\approx 1$ barn), whereas the fission cross section for thermal neutrons is $\approx 700$ barns. In order to achieve the required sensitivity to plutonium, the $14 \mathrm{MeV}$ source neutrons must be moderated to thermal or near thermal energies.

The energy of the neutrons produced by fission is $\approx 2 \mathrm{MeV}$. Each shielded detector bank is surrounded by low-energy neutron absorber material (i.e., cadmium and boron). As a result, the shielded detectors are not sensitive to the interrogation thermal neutrons but are sensitive to the higher energy fission neutrons. The moderation process, which takes neutron generator neutrons from $14 \mathrm{MeV}$ to thermal, involves several scattering events in the graphite liner of the PAN shield enclosure cavity and/or in the hydrogenous material of the waste matrix and shield walls. These scattering events occur over a period of time following the neutron generator pulse. At about $700 \mu \mathrm{s}$, the thermal neutron population is approaching a maximum and the epi-thermal neutron population is approaching a minimum. To take advantage of the optimal thermal to epi-thermal flux ratio, a detector time gate window is opened from $700 \mu \mathrm{s}$ to $2,700 \mu \mathrm{s}$. This time interval is referred to as the "early gate."

An active background count is taken by opening a second detector time gate window from $5,700 \mu s$ to $15,700 \mu$ s following each neutron generator pulse. During this time window the interrogation thermal neutrons will have died away and what remains are neutrons from spontaneous sources; e.g. cosmic neutrons, spontaneous fission neutrons, and $(\alpha, n)$ neutrons. This time interval is referred to as the "late gate." 
Each shielded detector bank, cavity flux monitor and barrel flux monitor has two scalars, one to accumulate counts from the early gate window and one to accumulate counts from the late gate window.

After completing an active mode acquisition, the following information is used to arrive at plutonium mass.

1. Shielded total early gate $(700 \mu \mathrm{s}-2,700 \mu \mathrm{s})$ count,

2. Shielded total late gate $(5,700 \mu \mathrm{s}-15,700 \mu \mathrm{s})$ count,

3. Cavity flux monitor early gate $(700 \mu \mathrm{s}-2,700 \mu \mathrm{s})$ count,

4. Cavity flux monitor late gate $(5,700 \mu \mathrm{s}-15,700 \mu \mathrm{s})$ count,

5. Barrel flux monitor early gate $(700 \mu \mathrm{s}-2,700 \mu \mathrm{s})$ count,

6. Barrel flux monitor late gate $(5,700 \mu \mathrm{s}-15,700 \mu \mathrm{s})$ count,

7. Number of neutron generator pulses.

\subsection{Data Analysis and Reported Pu Mass Selection}

SWEPP Assay System (SAS) Version 2.1 analysis code was used to produce the PAN plutonium mass values given in this report. A description of the analysis algorithms is given in the SAS code software requirements document (East et al., 1997) and will not be repeated in this report. As stated previously the PAN system does both an active mode assay and a passive mode assay. Therefore, there are two $\mathrm{Pu}$ mass values generally available to report; i.e., active $\mathrm{Pu}$ mass or passive $\mathrm{Pu}$ mass. The passive $\mathrm{Pu}$ mass however, is chosen from among the short-gate coincidence $\mathrm{Pu}$ mass, the long-gate coincidence Pu mass and the shift register coincidence Pu mass. So in fact, there are four Pu mass values generated in a PAN system assay; i.e., one from active mode and three from passive mode.

Ideally, all four mass values should agree. In most cases, they do not and so a decision has to be made as to which value is best in terms of accuracy and should be reported as the Pu mass. For each content code, an assessment is performed to determine generally whether the active mode or passive mode assay results should be used for that content code. The choices are: (1) active mode for all drums of that content code, (2) passive mode for all drums of that content code or (3) default to SAS code selection algorithm. In the default option, the decision between active and passive Pu mass is based on a threshold value of $5 \mathrm{~g}$ measured passive $\mathrm{Pu}$ mass; i.e., if the passive $\mathrm{Pu}$ mass is at or above $5 \mathrm{~g}$, the passive Pu mass will be reported. If, on the other hand, the passive Pu mass is less than $5 \mathrm{~g}$, the active mode $\mathrm{Pu}$ mass will be reported. If the "passive Pu mass" is chosen (either by option (2) or option (3) above), the shift register coincidence $\mathrm{Pu}$ mass will be used in almost all cases. If the shift register $\mathrm{Pu}$ mass is not available or is suspect, then either the short-gate coincidence mode Pu mass or the long-gate coincidence mode $\mathrm{Pu}$ mass will be reported as the passive Pu mass. The decision between these two is based on which has the smaller relative error.

For most sludge waste codes and certainly for the IDCs analyzed in this report, high $(\alpha, \mathbf{n})$ background and low plutonium concentration has led to the active mode Pu mass (i.e., Option 1), being specified as the reported Pu mass.

\subsubsection{U-235 correction to the active Pu mass determination}

In the original PAN calibration and analysis routines (Caldwell et al.,1985; Caldwell et al. 1986), the assumption was made that there is only weapons grade plutonium in the transuranic waste drums from Rocky Flats Plant. However, the addition of the SWEPP Gamma Ray Spectroscopy (SGRS) system 
quickly identified that uranium was also present and for some content codes U-235 is the dominant fissile nuclide. The SAS code version 2.1 (East et al., 1997) contains routines, which account for fission from $\mathrm{U}-235$ as well as fission from Pu-239 in the active mode assay. Therefore, uranium compensation can only be accomplished if the U-235 to Pu-239 mass ratio is passed over from the SGRS to the PAN prior to the commencement of the SAS analysis. 


\section{CALIBRATION OF THE PAN ASSAY SYSTEM}

The basic calibration of the PAN system is performed using standard sealed neutron sources in an empty waste drum. The original calibration was performed by LANL prior to delivery of the PAN system to the INEEL. Because of changes made to the detector electronics and the addition of the shift register coincidence system, a baseline calibration was repeated in June 1997 (Harker et al., 1997).

Listed below are the basic equations used by the PAN assay system to determine the mass assay values.

$\operatorname{Mass}_{\mathrm{A}}=\mathrm{C}_{\mathrm{A}} *[\text { (Net Total Shld Adj Rate / Net Flux Mon Rate) }- \text { Int. Bkgd }]_{\mathrm{A}} * \mathrm{CF}_{\mathrm{A}}{ }^{*} \mathrm{CF}_{\mathrm{I}}$

Mass $_{\mathrm{lg}}=\mathrm{C}_{\mathrm{lg}} *(\text { Net LG Coincidence Rate })_{\mathrm{P}} * \mathrm{CF}_{\mathrm{lg}} * \mathrm{CF}_{\mathrm{I}}$

Mass $_{\mathrm{sg}}=\mathrm{C}_{\mathrm{sg}} *(\text { Net SG Coincidence Rate })_{\mathrm{P}} * \mathrm{CF}_{\mathrm{sg}} * \mathrm{CF}_{\mathrm{I}}$

Mass $_{\mathrm{src}}=\mathrm{C}_{\mathrm{lg}} *(\text { Net SRC Coincidence Rate })_{\mathrm{P}} * \mathrm{CF}_{\mathrm{sg}} * \mathrm{CF}_{\mathrm{I}}^{*}(\mathrm{SRC}$ calibration adjustment $)$

where:

Net Total Shld Adj Rate $=$ Net total shielded adjusted rate,

Net Flux Mon Rate = Net flux monitor rate,

Int. Bkgd = Interrogation background,

Mass $_{\mathrm{A}}=\mathrm{Pu}$ mass as determined from the active mode,

Mass $_{\mathrm{lg}}=\mathrm{Pu}$ mass as determined by the long-gate coincidence mode,

Mass $_{\mathrm{sg}}=\mathrm{Pu}$ mass as determined by the short-gate coincidence mode,

Mass $_{\mathrm{src}}=\mathrm{Pu}$ mass as determined by the shift register coincidence mode,

$\mathrm{C}_{\mathrm{A}}, \mathrm{C}_{\mathrm{gg}}, \mathrm{C}_{\mathrm{sg}}$ are the base calibration coefficients for the active, long-gate coincidence, and shortgate coincidence modes, respectively,

$\mathrm{CF}_{\mathrm{A}}, \mathrm{CF}_{\mathrm{lg}}, \mathrm{CF}_{\mathrm{sg}}$ are the matrix correction factors for the active, long-gate coincidence, and shortgate coincidence modes, respectively,

SRC calibration adjustment is the factor applied to the long-gate base calibration coefficient to arrive at the SRC base calibration coefficient, and

$\mathrm{CF}_{\mathrm{I}}$ is the isotopic correction factor which takes into account that the $\mathrm{Pu}-240$ (passive mode) and $\mathrm{Pu}-239$ (active mode) mass fractions may be different from those used during the base calibration.

The effects of waste matrix, etc. on the system response were estimated during the original calibration series and an algorithm for determining the correction factors was developed by LANL. The correction factors were determined empirically using surrogate waste drums in which generic materials; e.g., vermiculite, boric acid, sand, and metal scraps were used to simulate the waste matrix. The basic assumption in the development of the simulated waste was that the matrix was uniform, the source distribution was uniform and that each waste drum was filled to near the volume capacity of the drum. Over the years, there have been small changes made to the correction factor algorithm, but the basic premises; i.e., uniform matrix and uniform source distributions, have not changed. Additional matrix effects resulting from violations of the uniformity assumptions are discussed in the next section. The presence of these effects is the reason a more thorough uncertainty analysis is required. 


\section{CONTRIBUTORS TO THE ASSAY UNCERTAINTIES}

This section describes sources of uncertainty in PAN measurements. It is the combined effects of these various contributors to errors in measurements that must be estimated in the uncertainty analysis.

\subsection{Base Calibration}

In the base calibration, the system response is measured for a well characterized neutron source (i.e., known neutron strength and elemental and chemical composition) at specified positions in an empty waste drum. There are three primary uncertainties associated with the base calibration. The first is the uncertainty for the source strength which includes any decay corrections which are applied and the number of neutrons produced per decay. The second is the uncertainty about the elemental and chemical composition of the neutron source material. The elemental and chemical composition can significantly affect the reported neutron source strength by producing an unknown number of neutrons produced by $(\alpha$, $n$ ) interactions in the source. The third uncertainty is the counting statistics associated with the base calibration data acquisition.

\subsection{Matrix and Source Effects}

In quantifying the estimates for systematic and random uncertainties, the major issue involves the validity of the uniform matrix and uniform source premise used in the PAN algorithm to the application of assaying a particular class of waste and what kind of errors are introduced as a result of making this assumption. Listed below are the specific ways that real waste may differ from the uniform matrix and uniform source premise.

1. Source isotopic/chemical composition effects.

2. Non-uniform matrix absorption.

3. Non-uniform matrix moderation.

4. Non-uniform source distribution.

5. Variations in source particle size.

6. Significant voids in the matrix.

7. Shadow shielding of one region by high neutron absorption in another region.

8. Waste elemental composition not addressed by the calibration routine.

\section{$4.3(\alpha, n)$ Source Interference}

In addition to the matrix and source introduced errors there are also uncompensated effects resulting from $(\alpha, n)$ reactions occurring in the waste. Since the $(\alpha, n)$ reactions only produce one neutron per reaction, the coincidence counting method in the passive mode should differentiate between neutrons produced by fission (more than one neutron per fission) and neutrons produced by $(\alpha, n)$ reactions. However, the coincidence counting method will have a contribution due to accidental or chance coincidences. There are standard techniques to correct the coincidence counting data for these spurious events and these techniques work well when the chance coincidence rate is small compared to the real coincidence rate. In those cases where the $(\alpha, n)$ source strength is clearly dominant over the fissile neutron source strength (i.e., the chance coincidence rate is dominant over the true coincidence rate) there is a very large uncertainty associated with the correction for chance coincidence events.

In addition, high count rates will also lead to counting losses which are not compensated for in the simple correction applied in the assay system analysis routine. For example, the standard corrections applied for 
counting losses are based on the assumption of random events and are not applicable to correlated events, as is the case in coincidence counting. The random event based corrections are valid when the correction is small but not when the counting loss is the same order of magnitude as the basic count rate. There are drums at RWMC where the neutron count rate is high enough that this circumstance applies. Under these situations the corrections are considered suspect and contribute significantly to the overall uncertainty of the measurement.

Thus ( $\alpha, n)$ interference and counting losses can be sources of significant uncertainties in the assay results. In fact, recent experience has indicated that these effects can be the dominant contribution to the uncertainty of the passive assay results. 


\section{UNCERTAINTY ANALYSIS APPROACH}

Evaluating the uncertainty of a complex nondestructive assay (NDA) system presents a number of challenges. While the intricate makeup of the system itself can make it difficult to adequately characterize performance, further complications occur when external factors must be considered. Such is the case with radioassay systems used to certify waste for shipment to the Waste Isolation Pilot Plant. The DOE requires that uncertainty evaluations for these systems include the effects of waste matrix parameters and other external factors having potential effects on system performance. Section 9 of the DOE's Transuranic Waste Characterization Quality Assurance Program Plan (DOE, 1996) requires performance goals be "achievable in the presence of backgrounds generated by alpha and gamma emitting sources and in the presence of interfering quantities of neutron and gamma absorbing and moderating material." In other words, the uncertainty characteristics of the system in its intended "real-world" application must be established.

Standard methods of uncertainty analysis, primarily based on propagation of errors, will almost always underestimate measurement uncertainty in real-world applications. This is because the uncertainties for the component parts are generally based on laboratory experiments on standard test materials. Such assessments are useful in that they provide a best case scenario of a system's performance capability. However, to the extent that they fail to include effects of external factors, they are insufficient to establish the quality of measurements achievable in a true operational setting. Factors not commonly considered in laboratory assessments of uncertainty include highly variable background levels or interference, operator effects (e.g., fatigue, level of training), and heterogeneity of materials being tested (matrix effects). The amount of variability inherent in some of these factors, especially matrix effects, makes them difficult or inappropriate to incorporate into analyses using standard methods.

When external factors such as matrix effects are expected to have significant influence on measurements, a key requirement for a study designed to assess the uncertainty of an NDA system is that the test items must be representative of those on which the system is intended to be used. Another way to think about uncertainty that focuses on the issue of representativeness is to consider the following question. If I select an item at random from a population of items of interest and measure it, what is the expected bias and precision error for that measurement? Stated in this manner, the importance of the representativeness of sampled items in the presence of matrix effects is clear. In a waste drum radioassay system for exarnple, results from an uncertainty evaluation using drums filled with a benign matrix cannot be legitimately used to infer the uncertainty for drums containing large quantities of shielding material such as lead.

Once uncertainty is considered from the viewpoint of the bias and precision of a randomly selected item from a population of interest, a general alternative method of uncertainty analysis emerges. This approach is a sampling and verification approach. For the PAN system, the sampling and verification approach is based on measuring a sample of real waste drums using both the PAN system and a confirmatory system and comparing the results. Under the assumption that the confirmatory data are unbiased, a regression analysis of the pairs of measurements yields estimates of both bias and precision of the PAN system for solidified aqueous sludge waste measurements.

The confirmatory measurement results for the solidified aqueous sludge waste drums were obtained from destructive radioassay of core samples from selected drums. A facility has been built at the INEEL for coring and sampling sludge waste drums. Originally developed for sampling for Resource Conservation Recovery Act (RCRA) listed hazardous constituents, core sampling plans were modified to include sampling for radioassay analysis as well. While the comparative drum core radioassay data contain a certain amount of uncertainty themselves, they are expected to be unbiased. So by comparing the rnean core sample results to the mean PAN results, the bias of the PAN system can be easily established. 
Furthermore, applying the proper variance component analysis will allow the standard deviation of errors to be estimated as well.

Uncertainty analyses for debris waste forms previously reported (e.g., Blackwood et al., 1997) also used sampling and verification methods. However, the debris waste evaluations were based on computer simulated measurements. The computer simulations for debris waste forms were necessitated by the lack of any practical method of verifying drum Pu content. For the sludge wastes, the existence of the coring facility made it quite practical to obtain actual confirmatory measurements.

As mentioned in the introduction, while the PAN system produces $\mathrm{Pu}$ mass estimates based on active mode and three types of passive mode counts, this report only addresses the uncertainty in the reported active mass value. The active mass value is always selected as the reported mass for sludge drums processed through the PAN system.

\subsection{Basic Steps in Performing the Uncertainty Analysis}

The basic steps used in performing the sampling and verification uncertainty analysis for the solidified aqueous sludge waste were as follows:

1. From IDCs $1,2,7,800,803,807$, select at least 100 drums for which PAN measurements are available from those being sent to the coring facility for RCRA evaluation.

2. Send samples from the cores for each of the selected drums to the INEEL Analytical Chemistry Laboratory for radiochemical assessment of TRU contents ( $\mathrm{Pu}, \mathrm{Am}$, and $\mathrm{U}$ isotopes).

3. Convert the chemical radioassay results $(\mathrm{pCi} / \mathrm{g})$ to the equivalent total drum contents (i.e., total grams of $\mathrm{Pu}$ ).

4. Compare the radiochemical analysis results with the PAN Pu measurement results for the same drums. From these data, estimates of both total bias and total precision for the PAN system sludge drum measurements can be obtained.

Details of the data collection and analysis activities are given in the following sections. 


\subsection{DATA}

The aqueous waste sludge drums selected to support the uncertainty analysis represent a subset of drums sampled to meet RCRA waste stream toxicity characterization requirements. The sampling plan for the RCRA analysis is described in detail in an internal engineering design file (Hailey, 1996). The RCRA sample procedure basically comprised a pseudo-random sample, i.e., random within the operational constraints of the facility (safety, accessibility, etc.). RCRA sampling was already in process when the decision was made to obtain radiochemistry samples as well. Once the procedures for the radiochemistry samples were determined, radiochemistry data on the remainder of the drums in the process were requested. Since the drums were sent to the coring facility in no particular order, the drums sent for radiochemistry analysis should be a representative sample of the aqueous waste sludge drums.

Of the total set of sludge drums selected for coring, 135 were initially identified to support the solidified aqueous waste uncertainty analysis using IDCs $1,2,7,800,803$, and 807 . However, radiochemistry analysis was not performed on 7 of the 135 drums. For most of these drums, the cores had already been obtained for the RCRA analysis when the request for the radiochemistry analysis was received at the laboratory. For one drum, sufficient core material on which to perform the radiochemistry analyses was not obtained during coring. Three additional drums were later eliminated from the uncertainty analysis due to lack of data associated with the PAN measurements, leaving 125 drums for the uncertainty analysis. The IDC breakdown for the 125 drums is given in Table 6-1.

Table 6-1. IDC breakdown for the 125 drums used in the uncertainty analysis.

\begin{tabular}{|r|r|}
\hline IDC & Number of drums \\
\hline 1 & 41 \\
\hline 2 & 8 \\
\hline 7 & 47 \\
\hline 800 & 16 \\
\hline 803 & 2 \\
\hline 807 & 11 \\
\hline Total & 125 \\
\hline
\end{tabular}

(Note: the IDC 807 waste included in this analysis is the solidified by-pass sludge waste from Building 374 at Rocky Flats. Generation of this waste began in March 1987. This 807 sludge is the same as the IDC 7 sludge generated using the by-pass system. This analysis does not include the IDC 807 cemented incinerator sludge from Building 771, generated from November 1985 to March 1987.)

\subsection{PAN Measurements}

Data on the PAN measurements for each of the 125 drums in the uncertainty analysis were obtained from the PAN historical database. Since the time the PAN measurements on these drums were obtained, changes in the PAN system have been made that affect the reported Pu mass values. These changes include installation of new electronic components, incorporation of the shift register passive counting mode, and revision of the zero-matrix calibration factors. Since it is not possible to remeasure the drums once the destructive core sampling has taken place, the original PAN measurement results for the 125 drums were adjusted to reflect the effects of the hardware and software changes. The proper adjustment was determined by comparing PAN measurements taken on a set of uncored sludge drums from the same content codes before and after the changes were implemented. Eleven such drums were selected. Their measured mass values before (old) and after (new) the PAN system changes were implemented are plotted in Figure 6-1. The reported numbers for the new PAN data are averages of results from two PAN 
measurement runs on each drum. For the old PAN data, only two drums had replicate measurements that were averaged. The remaining 9 drums had only one measurement.

Figure 6-1. Comparison of PAN system measurements before and after software and hardware system changes

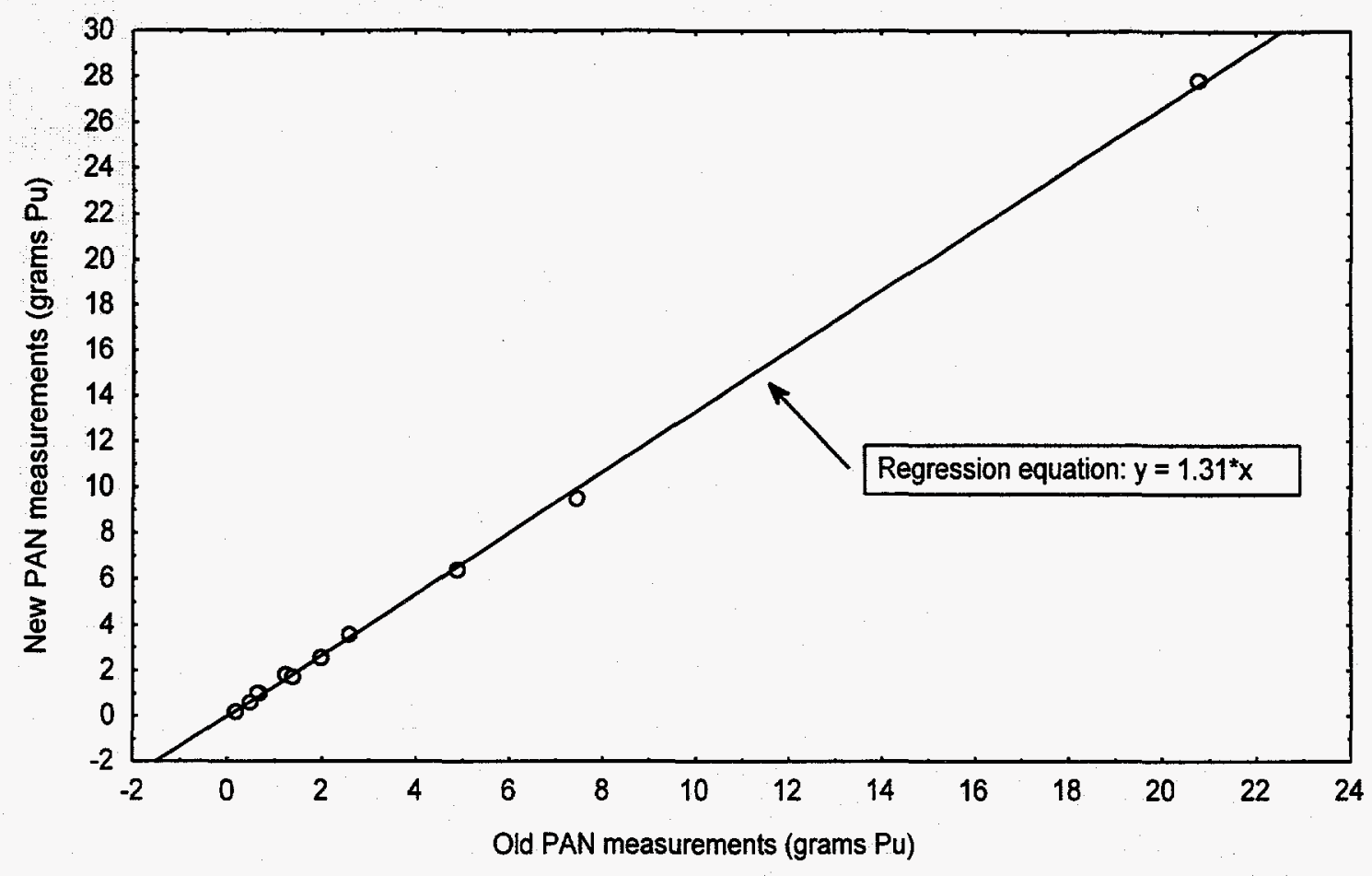

The new PAN data were regressed on the old PAN data using weighted least squares where the weights were based on the measured counting statistics error as described below. (In the cases where the averages of replicate measurements were used, the counting statistics errors were propagated to get the error for the average value.)

Let $y_{i}$ and $x_{i}$ be the observed new and old measurements (perhaps the average of two replicate measurements) for drum i. Assume that $y_{i}$ is distributed with mean $\mu_{y i}$ and standard deviation $\sigma_{y i}$. Similarly assume that $\mathrm{x}_{\mathrm{i}}$ is distributed with mean $\mu_{\mathrm{xi}}$ and standard deviation $\sigma_{\mathrm{xi}}$. A linear measurement model for the relationship between the old and new PAN results is then

$$
\mu_{y_{i}}=\beta_{0}+\beta_{1} \mu_{x_{i}} \text {. }
$$

Letting $\varepsilon_{\mathrm{yi}}=\mathrm{y}_{\mathrm{i}}-\mu_{\mathrm{yi}}$ and $\varepsilon_{\mathrm{xi}}=\mathrm{x}_{\mathrm{i}}-\mu_{\mathrm{xi}}$, the model can be restated in terms of the observed measurements as

$$
\begin{aligned}
y_{i} & =\beta_{0}+\beta_{1}\left(x_{i}-\varepsilon_{x_{i}}\right)+\varepsilon_{y_{i}} \\
& =\beta_{0}+\beta_{1} x_{i}+\varepsilon_{y_{i}}^{*}
\end{aligned}
$$

Where

$\varepsilon_{y_{i}}^{*}=\varepsilon_{y_{i}}-\beta_{1} \varepsilon_{x_{i}}$. 
Now the variance of $y_{i}$ is

$$
\begin{aligned}
\operatorname{var}\left(y_{i}\right) & =\operatorname{var}\left(\varepsilon_{y_{i}}^{*}\right) \\
& =\beta_{1}^{2} \sigma_{x_{i}}^{2}+\sigma_{y_{i}}^{2} .
\end{aligned}
$$

Since $\operatorname{var}\left(y_{i}\right)$ changes from one drum to another, a weighted least squares analysis is appropriate. The weights are estimates of $1 / \operatorname{var}\left(y_{i}\right)$. The squares of the measured counting statistics errors (or propagation

of those errors for cases where replicate measurements are involved) are used to estimate $\sigma_{x_{i}}^{2}$ and $\sigma_{y_{i}}^{2}$. Since $\beta_{1}$ must be estimated from the regression analysis itself, an iterative process is used. An initiall value of 1.0 is used for $\beta_{1}$ to calculate the weights. This produces a corresponding least squares estimate of $\beta_{1}$ in the regression. This new regression coefficient is used to recalculate the weights and the least squares analysis is repeated. These iterations continue until the estimates of $\beta_{1}$ converge.

The weighted least squares regression of the new PAN values on the old PAN values showed the intercept to be not statistically significant $(p=.31)$ so that term was eliminated from the model. The final regression equation was

new PAN Value $=1.31 *$ old PAN value.

The bulk of the difference in the two sets of values is attributable to the change made in the zero-matrix calibration coefficients for the PAN active mode measurements. (The ratio of the new to old coefficients is 1.26 .) Equation 6.5 fits the data very well, explaining $99.9 \%$ of the variability in the new PAN values. The standard error of the 1.31 adjustment factor is .037 .

Using Equation 6.5, the old PAN measurements for the 125 drums used in the uncertainty analysis were adjusted to reflect the measurements that would have been obtained after the changes in the PAN system had been implemented. These adjusted data values are used in all subsequent uncertainty calculations. The standard error of the adjustment factor is propagated along with other parameter estimate errors in calculating the final uncertainty value below.

\subsection{Radiochemical Analysis Results}

A minimum of two cores was planned from each of the 125 waste drums used in the uncertainty analysis. For one drum, multiple drilling attempts produced only one usable core. For one other drum, three cores were taken. Coring locations for each drum were selected at random from seven possible ports using a template placed over the drum prior to coring. Once the cores were removed from the drum, a slice of the recovered material, running the full length of the core, was obtained and homogenized. One aliquot was randomly sampled from each homogenized core slice and sent to the lab for analysis. An additional aliquot was obtained from some of the cores for QA purposes.

The analytical laboratory reported data for each drum sample in terms of activity (pCi per gram) for Am241, Pu-238, and Pu-239. However, the isotope labeled as Pu-239 by the laboratory actually represented the sum of $\mathrm{Pu}-239$ and $\mathrm{Pu}-240$. To get a single activity value for each drum, the $\mathrm{Pu}-239+\mathrm{Pu}-240$ data for samples from a particular drum were averaged first across replicates for each core and then across cores. Standard errors for the drum activity values were also calculated (based on the between core standard deviations in the drum). 
The drum activity values were converted to Pu mass quantities using the formula

$$
\mathrm{M}_{\mathrm{Pu}}=\frac{\mathrm{W} \times \mathrm{A} \times 10^{-12}}{\left(\mathrm{SA}_{\mathrm{Pu}-239}\right)\left(\mathrm{MF}_{\mathrm{Pu}-239}\right)+\left(\mathrm{SA}_{\mathrm{Pu}-240}\right)\left(\mathrm{MF}_{\mathrm{Pu}-240}\right)}
$$

where

$$
\begin{array}{ll}
\mathrm{M}_{\mathrm{Pu}} & =\text { drum plutonium mass }(\mathrm{g}) \\
\mathrm{W} & =\text { drum weight }(\mathrm{g}) \\
\mathrm{A} & =\text { average activity from the drum core samples }(\mathrm{pCi} / \mathrm{g}), \\
\mathrm{SA}_{\mathrm{Pu}-239} & =\mathrm{Pu}-239 \text { specific activity, } \\
\mathrm{MF}_{\mathrm{Pu}-239} & =\mathrm{Pu}-239 \text { mass fraction in weapons grade plutonium, } \\
\mathrm{SA}_{\mathrm{Pu}-240} & =\mathrm{Pu}-240 \text { specific activity, and } \\
\mathrm{MF}_{\mathrm{Pu}-240} & =\mathrm{Pu}-240 \text { mass fraction in weapons grade plutonium. }
\end{array}
$$

Errors in the mass fraction and drum concentration values were propagated to give the error in the total $\mathrm{Pu}$ mass value. (The drum mass was assumed to be known without error since its uncertainty is negligible compared to the other components.) These data were then compared to the PAN measurement values for total plutonium. 


\subsection{UNCERTAINTY ANALYSIS}

The total Pu mass as determined by radiochemistry analysis and the corresponding PAN measurements for each of the 125 drums in the uncertainty analysis are given in Appendix A. The data are plotted in Figure 7-1. The line of perfect agreement specified in the plot (i.e., the solid line) indicates where the data would fall if there were no measurement bias or precision error. The second line in the plot (i.e., the dashed line) is a regression line fit to the data using the method described below. The regression line indicates the degree of bias in the PAN system. That the regression line (and most of the data) fall above the line of perfect agreement indicates that the PAN Pu measurements are biased low compared to the confirmatory radiochemistry results. The degree of scatter in the points about the regression line is an indicator of the degree of precision in the measurements. Since both the PAN system and radiochemistry data are subject to precision error, the precision of the PAN system must be estimated by the decomposition of variance components in the regression model. The appropriate treatment of the variance components is determined by consideration of measurement models in the next section.

Figure 7-1. Comparison of PAN and radiochemistry Pu measurement results

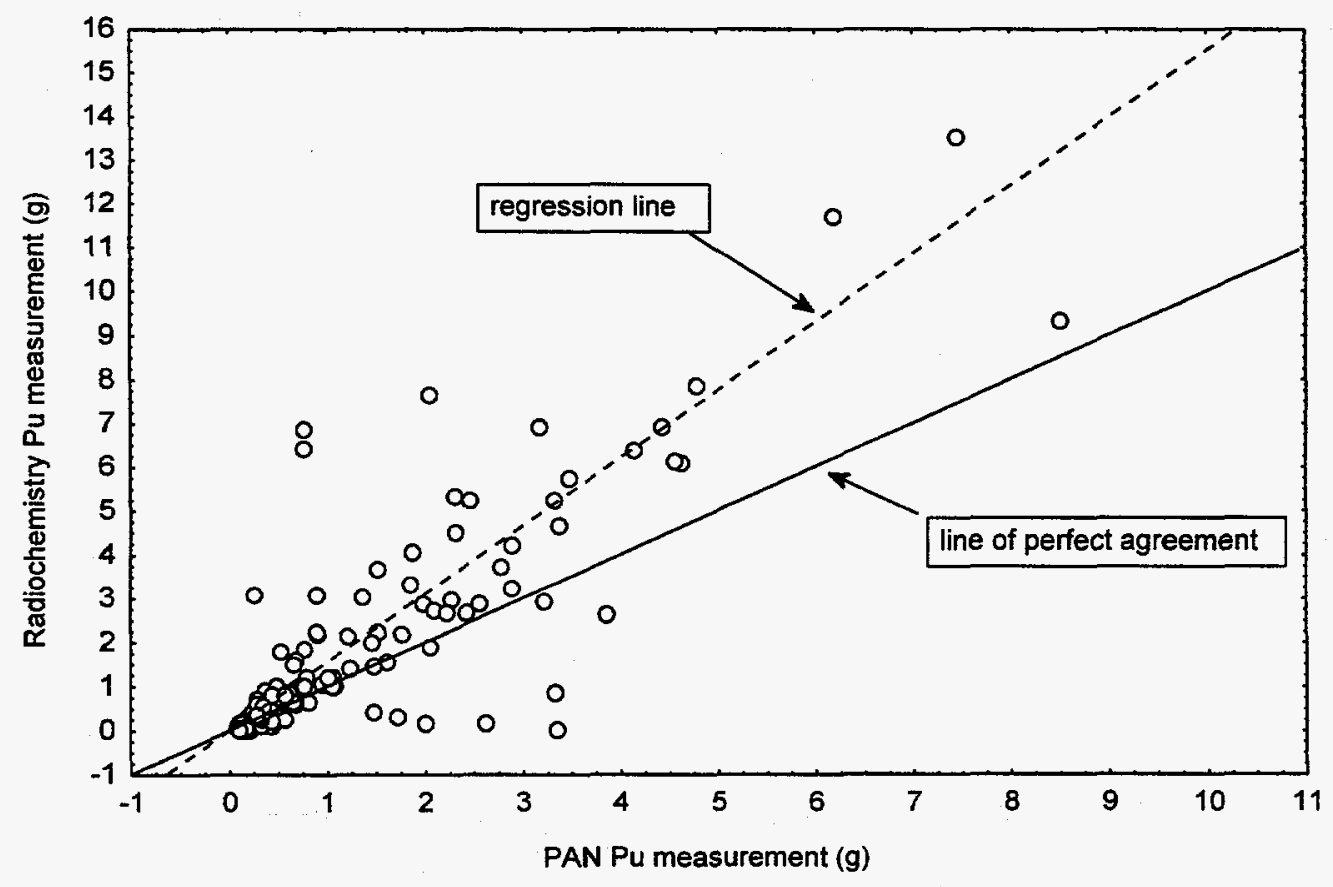

\subsection{Measurement Models}

Let $\xi_{\eta}$ be the theoretical mean of the PAN measurements of all drums in the waste population of interest that contain $\eta \mathrm{g} \mathrm{Pu}$. If the PAN system is unbiased, $\xi_{\eta}=\eta$. If there is constant and/or relative bias in the PAN system then the relevant model is

$\eta=\alpha+\beta \xi_{\eta}$

where $\alpha$ is the constant bias effect and $\beta$ is the relative bias effect. If $\alpha \neq 0$ and $\beta=1$, then there is a constant bias in the measurement system. If $\alpha=0$ and $\beta \neq 1$, then there is constant relative bias in the 
system. It is also possible to have both constant and relative bias terms at the same time. To estimate the bias terms as well as address the precision error in the PAN system, we need to compare the measured Pu values from the PAN system and the radiochemistry data.

The observed PAN measurement for a randomly selected drum from those containing $\eta \mathrm{g} P u$ is equal to the theoretical mean $\xi_{\eta}$ plus random errors due to matrix effects and counting statistics. That is, if $x_{i}$ is the PAN measurement for drum $i$ and $\eta_{i}$ the true Pu value for drum $i$ then

$\mathrm{x}_{\mathrm{i}}=\xi_{\eta \mathrm{i}}+\delta_{\mathrm{mi}}+\delta_{\mathrm{ci}}$

where $\delta_{\mathrm{mi}}$ is the matrix effect of drum $i, \delta_{\mathrm{ci}}$ is the counting statistics error from the PAN system, and

$\mathrm{E}\left(\delta_{\mathrm{mi}}\right)=\mathrm{E}\left(\delta_{\mathrm{ci}}\right)=0$

$\operatorname{Var}\left(\delta_{\mathrm{mi}}\right)=\sigma_{\mathrm{mi}}^{2}$

$\operatorname{Var}\left(\delta_{\mathrm{ci}}\right)=\sigma_{\mathrm{ci}}^{2}$

$E($.$) and \operatorname{Var}($.$) are the expected value and variance of the indicated terms. The i$ in the subscript of the $\sigma^{2}$ terms indicates that the variance values can change from drum to drum. (Below we show that the error variances can be modeled as functions of the measured Pu quantity in the drum.)

Since the radiochemistry data are unbiased but measured with error, the measured radiochemistry value for drum $i$ is the true value plus a random measurement error term, i.e.,

$y_{i}=\eta_{i}+\delta_{r i}$

$\mathrm{E}\left(\delta_{\mathrm{ri}}\right)=0$

$\operatorname{Var}\left(\delta_{\mathrm{ri}}\right)=\sigma_{\mathrm{r}}^{2}$.

The measurement error in the radiochemistry value for drum $i, \delta_{\text {ri }}$, comprises all the error in the radiochemistry data (e.g., analytic error and core to core variability in the radiochemistry measurements).

A measurement model relating the radiochemistry data to the PAN data from which bias and precision estimates can be obtained via regression is created by first replacing the $\eta_{i}$ term in Equation 7.6 with Equation 7.1:

$y_{i}=\alpha+\beta \xi_{\eta i}+\delta_{i i}$

Next, solve for $\xi_{\eta i}$ in Equation 7.2 and substitute into Equation 7.9 to get

$$
\begin{aligned}
y_{i} & =\alpha+\beta\left(x_{i}-\delta_{m i}-\delta_{\mathrm{ci}}\right)+\delta_{r i} . \\
& =\alpha+\beta\left(x_{i}\right)+\delta_{r i}-\beta\left(\delta_{m i}+\delta_{c i}\right) \\
& =\alpha+\beta\left(x_{i}\right)+\varepsilon_{i}
\end{aligned}
$$

where 
$\varepsilon_{\mathrm{i}}=\delta_{\mathrm{ri}}-\beta\left(\delta_{\mathrm{mi}}+\delta_{\mathrm{ci}}\right)$

The model in Equation 7.10 is in terms of the observed PAN and radiochemistry measurements so estimates of $\alpha$ and $\beta$ can be obtained using statistical regression analysis techniques. Since the residual values (the differences between actual and predicted $y_{j}$ values) from this regression are estimates of the $\varepsilon$ values, the residuals, along with additional information, can be used to obtain precision component estimates.

\subsection{Bias}

A regression analysis was performed to estimate the parameters $\alpha$ and $\beta$ in Equation 7.10. Since the variability of the data increases with increasing Pu quantity (see Figure 7-1), a weighted least squares analysis was performed (Neter and Wasserman, 1974). Ideally, the weights used in the analysis should be $1 / \sigma_{x}^{2}$ where $\sigma_{x}$ is the standard deviation of $\varepsilon$ for a given value of $x$. True values of $\sigma_{x}$ are unknown but can be estimated by estimating $\varepsilon$, since $s_{x}=|\varepsilon|$ is an estimate of $\sigma_{x}$.

Equation 7.11 cannot be used to estimate $\varepsilon$. While we have estimates of two of the needed compcinents for each drum, the radioassay measurement error $\delta_{\mathrm{ri}}$ and the PAN counting statistics error $\delta_{\mathrm{ci}}$, independent estimates of the remaining two components $\beta$ and $\delta_{\mathrm{mi}}$ are not available. Hence, both the weights and the regression coefficients were estimated by the following iterative method.

\subsubsection{Weighted least squares estimation}

In the first iteration of the weighted least squares analysis the weights were all set to 1.0 (i.e., an ordinary least squares analysis was performed). To obtain new weight estimates, the estimated values of $\alpha$ and $\beta$ from this regression were first substituted into Equation 7.10. Solving for $\varepsilon_{\mathrm{i}}$ and taking absolute values gives the new $s_{x}$ value for each of the $x_{i}$ data points. While each of the $s_{x}$ values is an estimate of the corresponding $\sigma_{x}$ value, taken individually they are highly inaccurate estimates. Better estimates of the weights can be obtained by considering the whole set of $s_{x}$ estimates in terms of their relationship to the PAN measured mass values. A scatterplot of the $s_{x}$ values and the measured PAN mass values showed the relationship to be linearly increasing on a log-log scale. A numerical estimate of this increasing relationship was obtained by regressing the logarithms of the $s_{x}$ values on the logarithms of the PAN mass values. New estimated values for $s_{x}$ obtained from this regression equation were then squared and, after taking reciprocals, used as weights in the second iteration of the weighted least squares analysis. This yielded new estimates of $\alpha$ and $\beta$, from which new estimates of the $\varepsilon_{\mathrm{i}}$ could be obtained. This iterative process was repeated until the estimates of $\alpha$ and $\beta$ and $\varepsilon_{\mathrm{i}}$ did not change in the first three significant digits.

The final equation used for calculating $s_{x}$ and hence the weights in the final iteration of the weighted least squares analysis was

$\mathrm{s}_{\mathrm{x}}=.365$ (PAN Pu mass) $^{931}$.

The data from which this equation was obtained via regression analysis are plotted in Figure 7-2. 
Figure 7-2. Final weight function used in the weighted least squares

regression of the radiochemistry results on the PAN results.

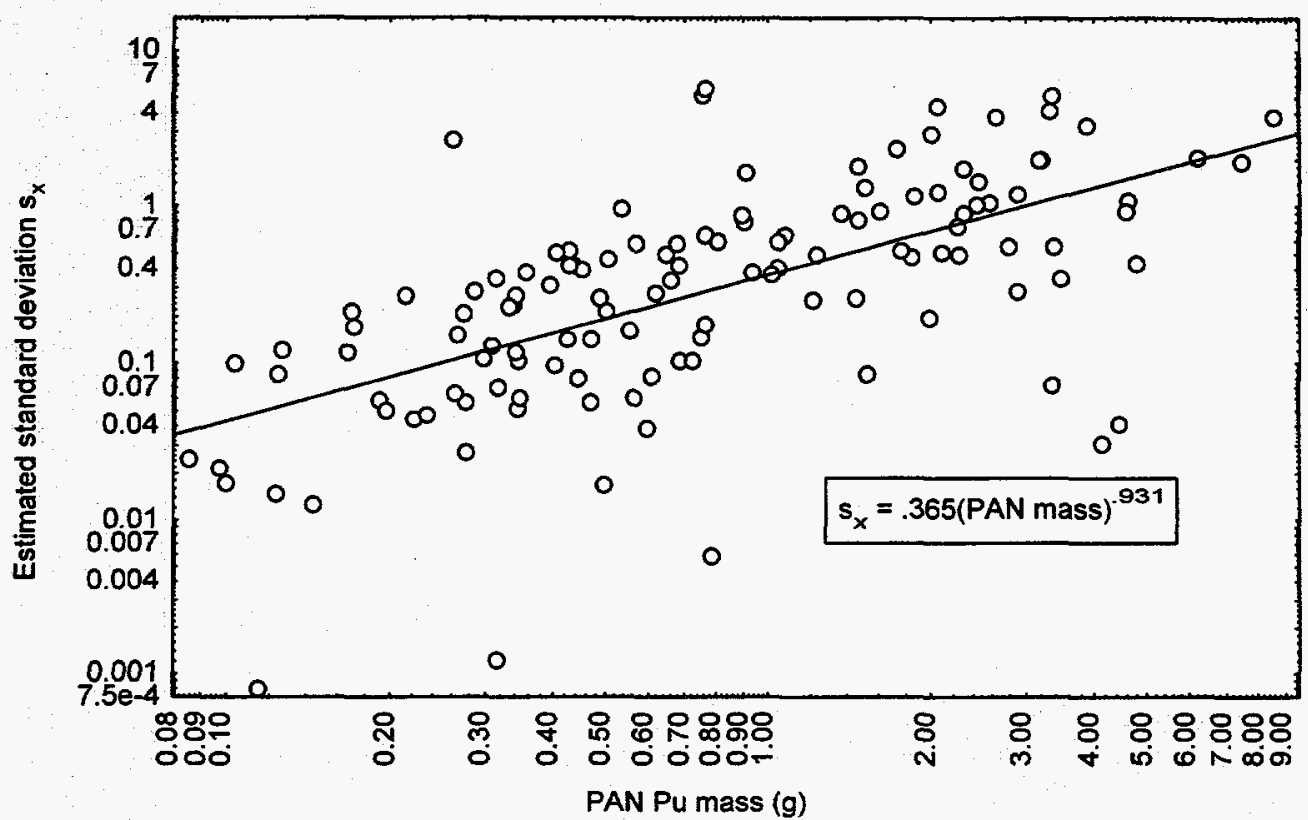

\subsubsection{Weighted Least Squares Analysis Results}

The weighted least squares analysis for the measurement model in Equation 7.10 produced an estimate for $\alpha$ that was not significantly different from zero, so that term was dropped from the model. The estimated value for $\beta$ is $\hat{\beta}=1.55$ with a standard error of .128 . To check the adequacy of a linear model for the data, a quadratic term was also tested. The quadratic term was not statistically significant $(p=.48)$ indicating the linear model is sufficient to represent the data. Therefore, the only applicable bias term is the relative bias term $\beta$. That is, the model relating the PAN system measurements to the radiochemistry results is simply

$y=\beta x$

which is estimated as

$\mathrm{y}=1.55 \mathrm{x}$

Under the assumption that the radiochemistry data are unbiased, Equation 7.14 becomes an expression for quantifying the bias in the PAN system.

While the standard error of $\hat{\beta}$ from the weighted least squares regression is .128 , the total uncertainty in estimating $y$ also includes the uncertainty in the correction factor applied to the PAN data prior to the analysis as described in Section 6.1. The uncertainty in the correction factor is however not included in the regression standard error of .128 for $\hat{\beta}$. How that error should be propagated into the error for $\hat{\beta}$ is described below. 
First, note that $\mathrm{x}$ in Equation 7.13 is the estimated "New PAN value" obtained from the "Old PAN value" via the correction factor derived in Section 6.1. Thus Equation 7.13 can be restated as

$y=\beta^{*}$ New PAN value

$=\beta^{*} \delta *$ Old PAN value

$=\gamma^{*}$ Old PAN value

where $\delta$ is the correction factor from Section 6.1 and $\gamma=\beta^{*} \delta$. Performing a regression to estimate $\gamma$ in Equation 7.17 is the same as the regression for estimating $\beta$ in Equation 7.13 , except for the constant correction factor $\delta$ applied to the data. The estimated correction factor was $\hat{\delta}=1.31$ so the regression estimate of $\gamma$ in Equation 7.17 is

$$
\begin{aligned}
\hat{\gamma} & =\hat{\beta} * \hat{\delta} \\
& =1.55^{*} 1.31 \\
& =2.03 .
\end{aligned}
$$

The standard error of $\hat{\gamma}$ is $1.31^{*} .128=.168$. (The error in the estimate of 1.31 is not applied here because we are just backing out the constant to get the results in terms of the old rather than new PAN data.)

From Equations 7.18 we can get a new expression for $\hat{\beta}$ from which its total uncertainty, including that due to the correction factor $\hat{\delta}$, can be calculated. We have

$$
\hat{\beta} \quad=\hat{\gamma} / \hat{\delta}
$$

Applying standard propagation of errors to Equation 7.21, the standard error of $\hat{\beta}$ is calculated as

$$
s_{\hat{\beta}}=\sqrt{\left(\frac{\hat{\gamma}}{\hat{\delta}}\right)^{2}\left(\left(\frac{s_{\hat{\gamma}}}{\hat{\gamma}}\right)^{2}+\left(\frac{s_{\hat{\delta}}}{\hat{\delta}}\right)^{2}\right)},
$$

where $s_{\hat{\gamma}}$ and $s_{\hat{\delta}}$ are the estimated standard errors for $\hat{\gamma}$ and $\hat{\delta}$ obtained from the previous regression results. Substituting in the values for the variables in Equation 7.22 gives $s_{\hat{\beta}}=.136$.

Thus, under the assumption that the radiochemistry data are unbiased, applying a correction factor of 1.55 to PAN system measurements of aqueous sludge waste will yield an expected bias of zero with standard error .136. An approximate $95 \%$ confidence interval for the true relative bias of PAN measurements after the adjustment is $(-27 \%, 27 \%)$. 


\subsection{Matrix Precision Error}

An expression for calculating matrix precision error can be found by calculating the variance of both sides of Equation 7.10 and solving for the matrix precision error. This gives the precision error as

$\sigma_{\mathrm{mi}}=\sqrt{\frac{\sigma_{\varepsilon i}^{2}-\sigma_{\mathrm{i}}^{2}-\beta^{2} \sigma_{\mathrm{ci}}^{2}}{\beta^{2}}}$

where $\sigma_{\mathrm{\varepsilon i}}$ is the residual standard deviation (i.e., the standard deviation of the $\varepsilon$ values) and all the other terms are defined as before.

From Equation 7.23, estimates of the matrix precision error can be calculated using reported radiochemistry measurement error values to estimate $\sigma_{\text {ri, }}$ PAN reported counting statistics error values to estimate $\sigma_{c i}$, and estimates of $\beta$ and $\sigma_{\varepsilon i}$ produced in the weighted least squares regression above.

Matrix precision error is related to the mass of $\mathrm{Pu}$ in the drum, as can be seen in Figure 7-3, which plots the calculated matrix precision error terms as a function of the PAN Pu mass. A weighted least squares regression was applied to the matrix precision error data, resulting in the following formula for estimating the matrix precision error:

$s_{\mathrm{m}}=.40 *(\mathrm{PAN}$ Pu mass $)$.

(The data in Figure 7-3 are plotted using logarithmic scales for clarity. However, the weighted least squares regression was performed on data expressed in the original scales. Negative error estimates were set to zero for the analysis.)

Equation 7.24 indicates a relative matrix precision error of approximately $40 \%$. It should be noted that the regression fit is affected somewhat by several high uncertainty values. If the relative matrix precision error is calculated for each of the 125 individual drums the mean value is .43 , which agrees closely with the regression parameter estimate of .40 . However, the median relative uncertainty value is .16 , a considerably lower value.

\subsection{Total Precision Error}

Total precision error, $\sigma_{p}$, is comprised of the matrix precision error and the counting statistics error. For a given PAN measured mass, the total precision error (expressed as a standard deviation is) is

$$
\sigma_{p}=\sqrt{\sigma_{c}^{2}+\sigma_{m}^{2}}
$$

Substituting the values for the matrix error estimated in previous section gives the estimated total precision error as

$s_{p}=\sqrt{s_{c}^{2}+s_{m}^{2}}$

where $s_{c}$ is the PAN reported counting statistics error and $s_{m}$ is the matrix precision error. (For the analysis in this report, matrix precision error as calculated by Equation 7.23 is used. In general, Equation 7.24 must be used for PAN system reporting because the radiochemistry information needed for applying Equation 7.23 is not available.) 
Figure 7-3. Estimated matrix precision error as a function of PAN Pu mass

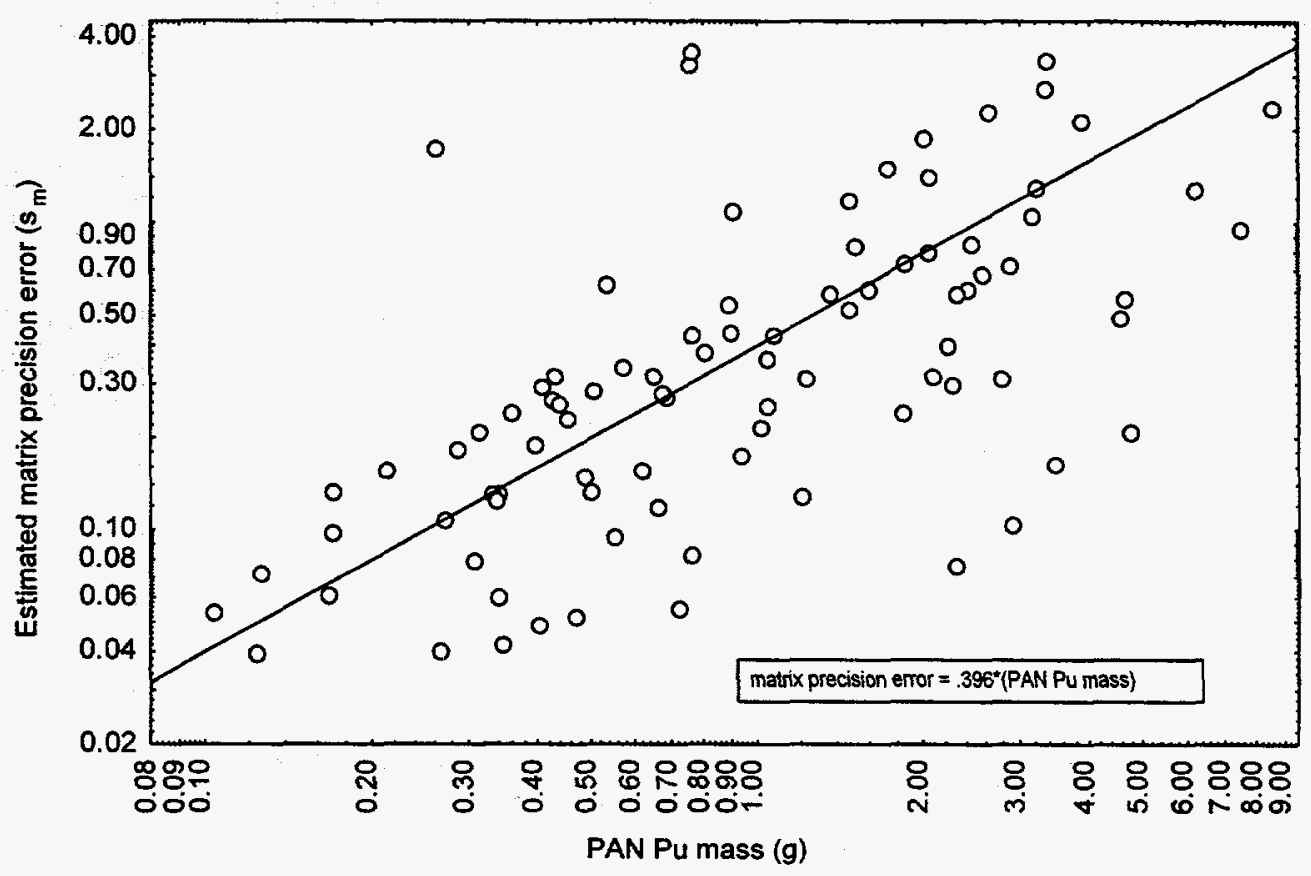

\subsection{Total Uncertainty}

To estimate the total uncertainty in a bias corrected PAN measurement for aqueous waste, consider propagation of error in the bias corrected Pu mass formula given in Equation 7.14. That equation was

$$
\begin{aligned}
\mathrm{y} & =\hat{\beta} \mathrm{x} \\
& =1.55 \mathrm{x}
\end{aligned}
$$

where $x$ is the PAN mass value before bias correction and $\hat{\beta}=1.55$ is the bias correction parameter determined by the weighted least squares analysis.

Acknowledging that both the measured Pu mass $x$ and $\hat{\beta}$ have associated precision error, applying standard propagation of errors to Equation 7.13 gives the estimated total uncertainty in a bias corrected measurement as

$$
s_{t}=\sqrt{1.55^{2} s_{p}^{2}+.136^{2} x^{2}}
$$

Where $s_{p}$ is the total precision error for the PAN measurement and .136 is the standard error of $\hat{\beta}$ as derived in Section 7.2.

The calculated total uncertainty in the bias corrected PAN Pu mass for each of the 125 aqueous waste drums is plotted in Figure 7-4. The uncertainty is plotted as a function of bias adjusted mass. A weighted least squares analysis was performed on the data to obtain a regression line for the data. (The data are 
plotted on a log-log scale for clarity. However, the regression was performed on the data in their original scales.) The fitted regression line is

total uncertainty $=.53$ (bias corrected PAN Pu mass)

indicating a total error approximately half the measured value. It should be noted that the regression fit is affected somewhat by several high uncertainty values. If the relative error is calculated for each of the 125 cases the mean value is .50 , which agrees closely with the regression parameter estimate of .53 . However, the median relative uncertainty value is .30 , a considerably lower value.

It should be noted that Equation 7.29 was included in this section as a means of summarizing the overall trend in total uncertainty. It should not be used to calculate and report total uncertainties for individual drum measurements. For individual drums the total uncertainty should be based on the specific drum measurements (i.e., the value from Equation 7.28).

Figure 7-4. Estimated total uncertainty as a function of bias corrected

PAN Pu mass

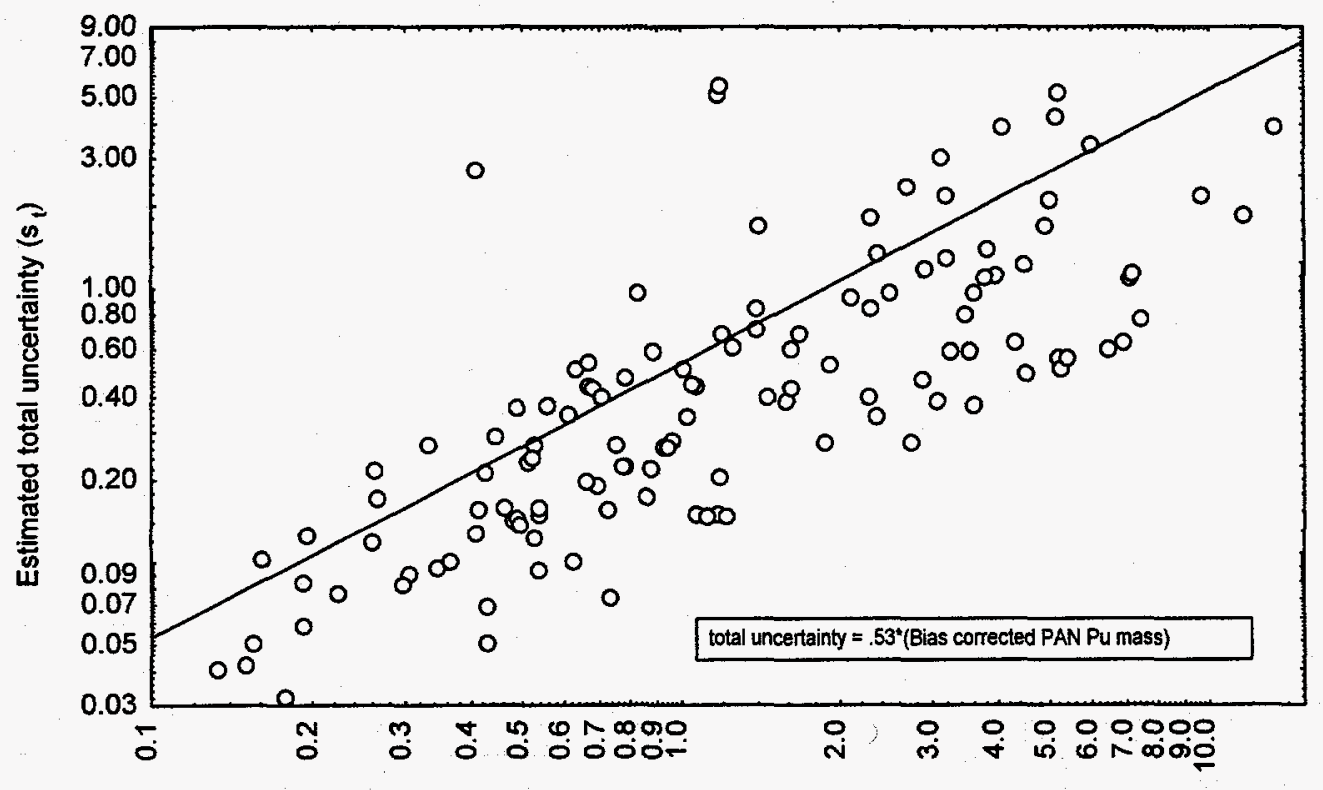

Bias corrected PAN Pu mass (g) 


\subsection{SUMMARY AND DISCUSSION OF RESULTS}

This report describes the results of a total uncertainty analysis of the PAN system's active mode measurements of aqueous sludge waste (item description codes 1, 2, 7, 800, 803, and 807). The uncertainty analysis was performed by comparing PAN system active mode results to radiochemistry results for 125 aqueous sludge drums. The radiochemistry data were obtained from core samples of the waste drums. Bias in the PAN system was estimated by regression of the radiochemistry results (assiumed to be unbiased) on the PAN measurements. Precision of the PAN measurements was assessed by examination of the variance components associated with the regression.

Analysis of the results for the 125 aqueous waste drums indicates that a bias correction multiplier of 1.55 should be applied to the PAN aqueous sludge measurements. Without this correction, the PAN measurements are biased low by $35 \%$ relative to the radiochemistry measurements.

The total uncertainty (expressed as a relative standard deviation) for the PAN measurement of a typical drum was found to be approximately $53 \%$, based on a regression analysis. However, the median value for the relative total uncertainty in the 125 cases was only $30 \%$. (For reporting purposes the total uncertainty for each drum will be determined individually and may be higher or lower than these typical values.)

Other follow-on calculations, primarily for producing certification reports for multiple drum units, are made in other documents and software related to the PAN system. These follow-on calculations make use of the results derived in this report. A table listing the various parameters required for those followon calculations is given in Appendix B.

Using the results of this evaluation we can make comparisons with quality assurance objectives (QAOs) as established in Table 9-1 of the TRU Waste Characterization Quality Assurance Program Plan (U. S. DOE, 1996). The parameter of interest in this table is "Total Bias" which is what has been evaluated in this report. QAOs are given for four different levels of total alpha activity or the equivalent quantity of weapons grade $\mathrm{Pu}$.

If the 1.55 estimated bias correction is applied to PAN measurements of aqueous sludge, the mean bias will be 0 . Equation 7.22 establishes the uncertainty in the total bias in the PAN Pu measurements. Based on the calculated standard error of .136, approximate $95 \%$ confidence interval bounds for the true $\mathrm{Pu}$ mass after the bias correction is applied are $0 \pm 27 \%$ of the actual Pu mass being measured. That is, the confidence bounds on the true value are $73 \%$ and $127 \%$.

Table 8-1 gives the bias limits for the four different Pu mass levels specified in the QAPP and compares them to the QAPP QAO limits. Since the bias correction is proportional to the measured Pu mass, the relative bounds on the PAN measurements are the same for all four compliance point levels. The relative bias error bounds for the PAN system compare favorably to the QAPP requirements.

In order to be critically safe, there is a safety limit requiring that the measured $\mathrm{Pu}$ mass plus 2 times its standard error must not exceed $200 \mathrm{~g}$. Based on the average total uncertainty calculations derived in this report the measured mass must be below approximately $97 \mathrm{~g}$ in order to meet this criterion for drums containing aqueous sludge waste. However, if the median total uncertainty value is used, the limit is increased to $125 \mathrm{~g}$. These summary mass values are only rough indicators of limits on shippable quantities as the actual upper limits will be calculated individually for each drum. In any case, the $200 \mathrm{~g}$ shipping limit is not likely to be an issue for sludge wastes as these drums typically contain only small quantities of $\mathrm{Pu}($ i.e., $<10 \mathrm{~g})$. 
Table 8-1. Comparison of QAPP QAO limits for bias to calculated $95 \%$ bounds for bias in PAN measurements of aqueous sludge.

\begin{tabular}{|c|c|c|c|c|}
\hline $\begin{array}{c}\text { Nominal } \\
\text { Compliance } \\
\text { Point } \\
(\mathrm{g} \mathrm{Pu})\end{array}$ & $\begin{array}{c}\text { Allowable } \\
\text { lower bound } \\
\text { for total bias } \\
(\%)\end{array}$ & $\begin{array}{c}\text { Allowable } \\
\text { upper bound } \\
\text { for total bias } \\
(\%)\end{array}$ & $\begin{array}{c}\text { PAN lower } \\
\text { bound for total } \\
\text { bias } \\
(\%)\end{array}$ & $\begin{array}{c}\text { PAN upper } \\
\text { bound for total } \\
\text { bias } \\
(\%)\end{array}$ \\
\hline .1 & 25 & 400 & 73 & 127 \\
\hline 1.0 & 35 & 300 & 73 & 127 \\
\hline 10 & 67 & 150 & 73 & 127 \\
\hline 160 & 67 & 150 & 73 & 127 \\
\hline
\end{tabular}




\section{REFERENCES}

Becker, G. K. (1993). "Drum Neutron Counter Chamber and Detector Configuration." EDF-RWMC606, Idaho National Engineering Laboratory.

Blackwood, L. G., Harker, Y. D., Meachum, T. R., Yoon, W. Y. (1997). "SWEPP PAN Assay System Uncertainty Analysis: Passive Mode Measurements of Graphite Waste." INEEL/EXT-97-00812, Idaho National Engineering and Environmental Laboratory.

Hailey, S. M. (1996). "Phase I Sampling Plan for TRU Waste Characterization Program." RWMC-909 (INEL-96/295), Idaho National Engineering and Environmental Laboratory.

Caldwell, J. T., Herrera, G. C., Hastings, R. D., Shunk, E. R., Kunz, W. E. (1985). "Calibration, Matrix Corrections, and Verification Activities with the Los Alamos Second Generation Combined Passive and Active Neutron Assay Systems." Los Alamos National Laboratory Program Technical Note, LA-Q2TN85-102, Los Alamos National Laboratory.

Caldwell, J. T., Hastings, R. D., Herrera, G. C., Kunz, W. E., Shunk, E. R. (1986). "The Los Alamos Second-Generation System for Passive and Active Neutron Assays of Drum-Size Containers," Los Alamos National Laboratory Technical Report, LA-10774-MS, Los Alamos National Laboratory.

East, L. V., Marwil, E. S., Matthews, S. D. (1997). "SWEPP Assay System Version 2.1 Software Requirements Specification," Idaho National Engineering Laboratory Technical Report, INEL/EXT-9700363, Idaho National Engineering Laboratory.

Harker, Y. D., Twedell, G., Hoffman, C. (1997). "Zero Matrix Calibration of the SWEPP PAN System." INEEL/INT-97-00866, Idaho National Engineering and Environmental Laboratory.

Neter, J. and Wasserman, W. (1974). Applied Linear Statistical Models: Regression, Analysis of Variance, and Experimental Designs, Homewood, IL: Richard D. Irwin, Inc.

U. S. Department of Energy (1996). "TRU Waste Characterization Quality Assurance Program Plan, Interim Change." CAO-94-1010. 


\section{APPENDIX A}

Table A-1. Radiochemistry and PAN Pu mass data for 125 aqueous sludge drums.

\begin{tabular}{|c|c|c|c|c|}
\hline \multirow[b]{2}{*}{ Barcode } & \multicolumn{2}{|c|}{ Radiochemistry Data } & \multicolumn{2}{|c|}{ PAN data } \\
\hline & Calculated mass & Standard error & Calculated mass & Standard error \\
\hline 513 & .21 & .02 & .11 & .05 \\
\hline 839 & .58 & .02 & .36 & .08 \\
\hline 887 & 4.50 & .06 & 1.76 & .09 \\
\hline 1080 & 7.86 & .04 & 3.65 & .18 \\
\hline 2006 & .11 & .01 & .07 & .03 \\
\hline 2212 & 3.10 & .06 & .20 & .07 \\
\hline 2923 & 1.07 & .01 & .71 & .17 \\
\hline 3042 & .61 & .01 & .34 & .12 \\
\hline 3227 & 1.21 & .03 & .80 & .07 \\
\hline 3268 & 1.22 & .04 & .60 & .05 \\
\hline 3325 & .30 & .02 & .17 & .06 \\
\hline 3344 & 3.10 & .23 & .69 & .04 \\
\hline 3512 & .06 & .00 & .16 & .08 \\
\hline 3546 & .07 & .00 & .10 & .04 \\
\hline 3760 & 2.87 & .16 & 1.51 & .13 \\
\hline 3932 & 5.74 & .17 & 2.65 & .11 \\
\hline 3963 & .13 & .01 & .33 & .14 \\
\hline 3966 & .12 & .01 & .31 & .15 \\
\hline 3986 & .26 & .00 & .20 & .09 \\
\hline 4219 & 1.00 & .01 & .82 & .06 \\
\hline 4221 & 2.13 & .15 & .92 & .04 \\
\hline 4237 & .25 & .01 & .33 & .10 \\
\hline 4344 & .14 & .00 & .13 & .05 \\
\hline 4410 & 1.54 & .02 & 1.22 & .06 \\
\hline 4456 & .31 & .02 & .39 & .11 \\
\hline 5049 & 5.27 & .58 & 1.87 & .10 \\
\hline 5398 & .20 & .07 & 2.00 & 1.03 \\
\hline 5427 & .42 & .13 & .24 & .08 \\
\hline 5695 & 4.66 & .60 & 2.57 & .15 \\
\hline 5698 & .31 & .03 & .35 & .12 \\
\hline 5703 & .31 & .01 & .44 & .15 \\
\hline 5735 & .18 & .00 & 1.53 & .22 \\
\hline 5738 & .29 & .01 & .30 & .08 \\
\hline 5766 & .14 & .01 & .24 & .08 \\
\hline 6276 & 1.02 & .12 & .57 & .07 \\
\hline 6435 & .48 & .00 & .26 & .09 \\
\hline 6439 & .49 & .00 & .24 & .09 \\
\hline 6711 & .18 & .00 & .09 & .02 \\
\hline 6729 & 1.45 & .10 & 1.12 & .10 \\
\hline 6782 & .17 & .08 & .07 & .03 \\
\hline 6836 & .65 & .01 & .62 & .07 \\
\hline 7085 & 2.64 & .17 & 2.94 & .12 \\
\hline 7832 & .29 & .02 & .26 & .09 \\
\hline 8344 & 3.34 & .29 & 1.40 & .07 \\
\hline 8355 & .79 & .21 & .36 & .02 \\
\hline 8371 & .94 & .08 & .27 & .02 \\
\hline 8473 & 1.86 & .03 & .58 & .02 \\
\hline 8569 & .35 & .05 & .23 & .10 \\
\hline 10534 & 5.25 & .42 & 2.54 & .22 \\
\hline 10549 & 1.03 & .11 & .80 & .11 \\
\hline 10604 & .45 & .02 & 1.12 & .13 \\
\hline 10788 & .68 & .09 & .47 & .08 \\
\hline 10859 & 1.40 & .07 & .93 & .08 \\
\hline
\end{tabular}




\begin{tabular}{|c|c|c|c|c|}
\hline \multirow[b]{2}{*}{ Barcode } & \multicolumn{2}{|c|}{ Radiochemistry Data } & \multicolumn{2}{|c|}{ PAN data } \\
\hline & Calculated mass & Standard error & Calculated mass & Standard error \\
\hline 10931 & 9.37 & .96 & 6.49 & .37 \\
\hline 11359 & .56 & .03 & .38 & .04 \\
\hline 11412 & .64 & .01 & .52 & .05 \\
\hline 11450 & 2.88 & .17 & 1.95 & .14 \\
\hline 11761 & 3.25 & .42 & 2.19 & .10 \\
\hline 11960 & .36 & .02 & .15 & .05 \\
\hline 12287 & 6.08 & .53 & 3.52 & .23 \\
\hline 12756 & 2.73 & .01 & 1.59 & .10 \\
\hline 12873 & .43 & .06 & .26 & .03 \\
\hline 13146 & 13.55 & 1.25 & 5.68 & .26 \\
\hline 13423 & .35 & .04 & 1.32 & .07 \\
\hline 13522 & 4.21 & .03 & 2.21 & .16 \\
\hline 13613 & 2.94 & .06 & 2.45 & .17 \\
\hline 13672 & .96 & .03 & .52 & .08 \\
\hline 13887 & 6.13 & .30 & 3.48 & .31 \\
\hline 13941 & .73 & .01 & .22 & .02 \\
\hline 14031 & .02 & .00 & 2.56 & .16 \\
\hline 14454 & .36 & .07 & .15 & .06 \\
\hline 15700 & 2.20 & .41 & .68 & .08 \\
\hline 16069 & 1.20 & .09 & .78 & .09 \\
\hline 16257 & .17 & .01 & .08 & .03 \\
\hline 16428 & .26 & .04 & .26 & .11 \\
\hline 17876 & 6.95 & 1.19 & 2.41 & .09 \\
\hline 17917 & 6.40 & .81 & 3.16 & .14 \\
\hline 18152 & 3.03 & .02 & 1.04 & .06 \\
\hline 18333 & 7.68 & 3.94 & 1.56 & .10 \\
\hline 18657 & 2.20 & .67 & 1.34 & .09 \\
\hline 18806 & 1.02 & .04 & .55 & .03 \\
\hline 19656 & .90 & .10 & 2.53 & .07 \\
\hline 19664 & .05 & .01 & .13 & .04 \\
\hline 20033 & 3.75 & .25 & 2.12 & .09 \\
\hline 20178 & .24 & .03 & .33 & .07 \\
\hline 20353 & .29 & .01 & .25 & .07 \\
\hline 20370 & .18 & .01 & .09 & .02 \\
\hline 20377 & .41 & .02 & .18 & .04 \\
\hline 20724 & .11 & .03 & .09 & .04 \\
\hline 20749 & 6.92 & .57 & 3.38 & .13 \\
\hline 20757 & 6.46 & 1.40 & .58 & .27 \\
\hline 20903 & .09 & .02 & .13 & .05 \\
\hline 22506 & .70 & .04 & .42 & .04 \\
\hline 23561 & 11.69 & .40 & 4.73 & .22 \\
\hline 23717 & 4.07 & .25 & 1.42 & .11 \\
\hline 23821 & 1.62 & .37 & .52 & .06 \\
\hline 23938 & .37 & .02 & .21 & .04 \\
\hline 24148 & .53 & .05 & .31 & .03 \\
\hline 24498 & 1.93 & .01 & 1.56 & .07 \\
\hline 24670 & 1.81 & .15 & .41 & .05 \\
\hline 25211 & .35 & .02 & .24 & .03 \\
\hline 25265 & 3.01 & .01 & 1.73 & .13 \\
\hline 25328 & .06 & .01 & .08 & .04 \\
\hline 27343 & 1.01 & .04 & .58 & .08 \\
\hline 27455 & .41 & .02 & .26 & .04 \\
\hline 28018 & 1.51 & .03 & .50 & .04 \\
\hline 28633 & 3.66 & .02 & 1.15 & .10 \\
\hline 29793 & 6.89 & 1.27 & .58 & .09 \\
\hline 30186 & .34 & .01 & .20 & .06 \\
\hline 30341 & 2.28 & .29 & .68 & .06 \\
\hline
\end{tabular}




\begin{tabular}{|l|c|c|c|c|}
\hline \multirow{3}{*}{ Barcode } & \multicolumn{2}{|c|}{ Radiochemistry Data } & \multicolumn{2}{c|}{ PAN data } \\
\cline { 2 - 5 } & Calculated mass & Standard error & Calculated mass & Standard error \\
\hline 30362 & 1.02 & .02 & .37 & .08 \\
\hline 30475 & 2.27 & .14 & 1.16 & .17 \\
\hline 31114 & .64 & .02 & .21 & .08 \\
\hline 31124 & .79 & .03 & .38 & .14 \\
\hline 31130 & 2.71 & .05 & 1.84 & .30 \\
\hline 31164 & .81 & .02 & .32 & .12 \\
\hline 31165 & .89 & .08 & .46 & .12 \\
\hline 31166 & 1.99 & .02 & 1.11 & .23 \\
\hline 31168 & .86 & .08 & .46 & .16 \\
\hline 31169 & .69 & .07 & .51 & .18 \\
\hline 31175 & 2.70 & .11 & 1.70 & .28 \\
\hline 31237 & 5.35 & 1.75 & 1.76 & .11 \\
\hline 31560 & .60 & .00 & .26 & .07 \\
\hline 31580 & .81 & .11 & .43 & .13 \\
\hline 32674 & .40 & .00 & .21 & .02 \\
\hline
\end{tabular}




\section{APPENDIX B \\ TABLE OF PARAMETERS REQUIRED FOR FOLLOW-ON CALCULATIONS}

The table below lists parameter values and associated errors generated in this report that are used in subsequent calculations in the processing of PAN data for aqueous sludge waste (IDCs 1, 2, 7, 800, 803, and 807). The subsequent calculations performed are documented in the INEEL engineering design file (internal publication INEL/INT-97-00140) titled "Use of SWEPP Assay System Version 2.1 Analysis Results to Produce Multiple Drum Certification Reports." Parameter names listed are those used in the engineering design file.

\begin{tabular}{|l|l|c|c|}
\hline \multicolumn{1}{|c|}{ Parameter Name } & \multicolumn{1}{|c|}{ Description } & Value & Error \\
\hline $\mathrm{AB}_{\mathrm{m}}$ & $\begin{array}{l}\text { Active mass bias correction } \\
\text { coefficient (intercept) }\end{array}$ & 0 & 0 \\
\hline $\mathrm{AB} 1_{\mathrm{m}}$ & $\begin{array}{l}\text { Active mass bias correction } \\
\text { coefficient (slope) }\end{array}$ & 1.55 & 0 \\
\hline $\mathrm{Cov}(\mathrm{AB} 0, \mathrm{AB} 1)$ & Covariance of $\mathrm{AB} 0_{\mathrm{m}}$ and $\mathrm{AB1} 1_{\mathrm{m}}$ & 0 & $-^{*}$ \\
\hline $\mathrm{AA} 0$ & $\begin{array}{l}\text { Active matrix precision coefficient } \\
\text { (intercept) }\end{array}$ & 0 & 0 \\
\hline $\mathrm{AA} 1$ & $\begin{array}{l}\text { Active matrix precision coefficient } \\
\text { (slope) }\end{array}$ & .40 & $-^{*}$ \\
\hline $\mathrm{AA} 2$ & $\begin{array}{l}\text { Active matrix precision coefficient } \\
\text { (quadratic) }\end{array}$ & 0 & 0 \\
\hline
\end{tabular}

*not required

(Note: the IDC 807 waste included in this analysis is the solidified by-pass sludge waste from Building 374 at Rocky Flats. Generation of this waste began in March 1987. This 807 sludge is the same as the IDC 7 sludge generated using the by-pass system. This analysis does not include the IDC 807 cemented incinerator sludge from Building 771, generated from November 1985 to March 1987.) 